\title{
Cálculo do Fator $Q$ de Combinações de Dipolos Elétricos e Magnéticos Infinitesimais com Diferentes Amplitudes, Fases e Orientações
}

\author{
Karlo Q. da Costa e Victor A. Dmitriev
}

\begin{abstract}
Resumo-Este trabalho apresenta uma análise do fator $Q$ de radiação de diferentes combinações de dipolos elétricos e magnéticos infinitesimais localizados no mesmo ponto no espaço livre. São considerados e comparados os casos de combinações de um dipolo elétrico e um dipolo magnético com momentos de dipolo com diferentes orientações, fases e amplitudes entre si. É mostrado que o mínimo fator $Q$ de radiação só é alcançado quando as amplitudes dos momentos de dipolo elétrico $\left(p_{e}\right)$ e magnético $\left(p_{m}\right)$ satisfazem a relação $\left|p_{m}\right|=Z_{0}\left|p_{e}\right|$ independentemente de suas orientações espaciais e fases relativas, onde $Z_{0}$ é a impedância característica do espaço livre.
\end{abstract}

Palavras-Chave-Fator $Q$ de radiação, dipolos elétricos e magnéticos infinitesimais, combinações de dipolos, mínimo $Q$.

\begin{abstract}
This work presents an analysis of the radiation $Q$ of different combinations of infinitesimal electric and magnetic dipoles localized at the same spatial point in free space. The combination cases of one electric dipole and one magnetic dipole with moments that possess different magnitudes, phases and orientations are analytically investigated and compared. It is shown that the minimum radiation $Q$ is achieved when the magnitudes of the dipoles moments electric $\left(p_{e}\right)$ and magnetic $\left(p_{m}\right)$ satisfy the equation $\left|p_{m}\right|=Z_{0}\left|p_{e}\right|$ independently of their relative spatial orientations and phases, where $Z_{0}$ is the characteristic impedance of the free space.
\end{abstract}

Index Terms-Radiation $Q$, infinitesimal electric and magnetic dipoles, combinations of dipoles, minimum $Q$.

\section{INTRODUÇÃO}

Com o avanço das comunicações móveis, torna-se cada vez maior o interesse por antenas que possuem pequenas dimensões e banda larga. Estes tipos de antenas possibilitam a construção de terminais móveis, por exemplo, telefones celulares, cada vez menores e com maior taxa de transmissão de informação (dados, voz, vídeos). Da teoria básica sobre antenas, sabe-se que quanto menor as dimensões do radiador em termos de comprimento de onda $\lambda$, menor será sua largura de banda e resistência de radiação, sendo que a redução deste último parâmetro implica em menor eficiência de radiação [1]. É devido a este comportamento geral que o projeto de antenas pequenas e de banda larga torna-se uma tarefa não trivial.

Karlo Q. da Costa e Victor A. Dmitriev, Departamento de Engenharia Elétrica e de Computação - DEEC, Universidade Federal do Pará, Belém/PA, Brasil, E-mails: karlo@ufpa.br, victor@ufpa.br. Este trabalho foi realizado com apoio financeiro do $\mathrm{CNPq}$.
Os primeiros estudos realizados sobre os limites fundamentais de antenas com relação as suas dimensões e largura de banda foram feitos por Wheeler [2] em 1947 e Chu [3] em 1948. Wheeler [2] utilizou a definição de fator de potência e obteve uma expressão deste parâmetro para duas antenas eletricamente pequenas representadas por um capacitor e um indutor. Estas antenas são equivalentes aos dipolos elétricos e magnéticos infinitesimais [1]. Chu [3] representou os campos radiados por uma antena qualquer sem perdas, localizada dentro de uma esfera fictícia de raio $a$, por uma série de ondas esféricas, e calculou através de circuitos equivalentes o fator de radiação desta antena em termos dos coeficientes da expansão em funções esféricas.

Estes dois autores obtiveram resultados similares sobre o efeito da redução das dimensões na largura de banda de uma antena qualquer. Uma de suas principais conclusões é que o mínimo fator de radiação que uma antena qualquer pode ter só é possível quando são radiados somente os modos fundamentais esféricos $\mathrm{TM}_{10}$ e $\mathrm{TE}_{10}$ com energias iguais. Estes modos são radiados pelos dipolos elétrico (DEI) e magnético (DMI) infinitesimais, respectivamente.

Collin e Rothschild [4] em 1964 confirmaram os resultados de Chu utilizando teoria de campos para calcular as energias envolvidas no cálculo do fator de radiação. Eles obtiveram expressões exatas para o cálculo do fator de radiação de antenas que radiam somente os modos $\mathrm{TM}_{\mathrm{nm}}$ ou $\mathrm{TE}_{\mathrm{nm}}$. Fante [5] em 1969 generalizou os conceitos de Collin e obteve uma expressão geral para o cálculo do fator de radiação de uma antena que radia qualquer modo esférico $\left(\mathrm{TM}_{\mathrm{nm}}+\mathrm{TE}_{\mathrm{nm}}\right)$. Este autor derivou também uma expressão exata para o mínimo fator de radiação que uma antena qualquer pode ter $\left(\mathrm{TM}_{10}+\mathrm{TE}_{10}\right)$. Esta expressão é aceita hoje em dia como limite fundamental. Em [6], McLean faz uma verificação destes limites fundamentais utilizando dipolos infinitesimais, e propõe um método mais simples para obtê-los. Os resultados obtidos também confirmam os anteriores. Trabalhos recentes sobre estes limites fundamentais, cálculo de fator de radiação, e aplicações de dipolos elétricos e magnéticos para aumento da largura de banda podem ser encontrados em [7]-[13].

Em [14] é apresentado uma análise de combinações dipolos elétricos e magnéticos infinitesimais com diferentes fases, amplitudes e orientações dos momentos de dipolos. O autor apresenta uma expressão para o fator de radiação somente para o caso onde $\left|p_{m}\right| \leq Z_{0}\left|p_{e}\right|$. 
No presente trabalho, é derivada uma expressão exata geral para o fator de radiação de combinações de dipolos elétricos e magnéticos infinitesimais alinhados e ortogonais, com diferentes fases e amplitudes dos momentos de dipolo. $\mathrm{O}$ método de cálculo do fator de radiação utilizado é o mesmo utilizado em [6]. São calculadas as energias elétrica e magnética reativas das combinações. Resultados da variação do fator de radiação em função das dimensões da antena, das fases e amplitudes dos momentos de dipolo são apresentados.

\section{DESENVOLVIMENTO TEÓRICO}

Esta seção apresenta o desenvolvimento teórico utilizado para o cálculo do fator de radiação $Q$ das duas combinações de dipolos elétricos e magnéticos infinitesimais considerados neste trabalho. A definição do fator de radiação $Q$ para antenas é [4]

$Q=\left\{\begin{array}{l}\frac{2 \omega W_{e}}{P_{r a d}} \text { se } W_{e}>W_{m} \\ \frac{2 \omega W_{m}}{P_{r a d}} \text { se } W_{m}>W_{e}\end{array}\right.$

onde $\omega$ é a freqüência angular ( $\mathrm{rad} / \mathrm{s}), W_{e}$ e $W_{m}$ são as energias reativas elétrica e magnética armazenadas ao redor da antena, respectivamente, e $P_{\text {rad }}$ é a potência média total radiada pela antena. Esta definição é para o caso de antenas sem perdas. Para o caso quando existem perdas $P_{\text {rad }}$ é a potência radiada mais a potência de dissipação. Nesta definição utiliza-se um elemento reativo externo de casamento nos terminais da antena para torná-la ressonante. Este elemento externo deve armazenar energia elétrica se $W_{e}<W_{m}$ ou magnética se $W_{e}<W_{m}$. Se o valor de $Q$ for alto $(Q>10)$, pode-se aproximar a largura de banda fracional $B$ como o inverso de $Q(Q \approx 1 / B)$. Se o valor de $Q$ for baixo, a impedância de entrada da antena irá variar lentamente com a freqüência e esta antena possuirá uma característica de banda larga [3].

O método utilizado aqui para o cálculo de $Q$ é o mesmo apresentado em [6]. Para exemplificar este procedimento de cálculo, é derivada também nesta seção a expressão do fator $Q$ do dipolo elétrico infinitesimal.

\section{A. Fator $Q$ do $D E I$}

Considera-se um dipolo elétrico infinitesimal no espaço livre com momento de dipolo elétrico $\bar{p}_{e}=p_{e} \bar{a}_{z}$ localizado na origem do sistema de coordenadas e direcionado ao longo do eixo $z$ positivo. Este dipolo representa uma antena com dimensões tal que o raio da menor esfera fictícia que circunscreve esta antena é $a$. Considera-se também o caso idealizado onde esta antena não possua perdas por condução e que não existe energia reativa armazenada no interior desta esfera, ou seja, $W_{e}=W_{m}=0$ para $r<a$ ( $r$ é a coordenada radial do sistema de coordenadas esféricas). Os campos eletromagnéticos radiados por este dipolo são [1]

$$
\begin{aligned}
& H_{\phi}=\frac{j k p_{e} \operatorname{sen} \theta}{4 \pi r}\left(1+\frac{1}{j k r}\right) e^{-j k r} \\
& E_{r}=\frac{Z_{0} p_{e} \cos \theta}{2 \pi r^{2}}\left(1+\frac{1}{j k r}\right) e^{-j k r} \\
& E_{\theta}=\frac{j Z_{0} k p_{e} \operatorname{sen} \theta}{4 \pi r}\left(1+\frac{1}{j k r}+\frac{1}{(j k r)^{2}}\right) e^{-j k r} \\
& H_{r}=H_{\theta}=E_{\phi}=0
\end{aligned}
$$

onde $k=\omega\left(\mu_{0} \varepsilon_{0}\right)^{1 / 2}$ constante de propagação do espaço livre, $\mu_{0}$ a permeabilidade do espaço livre, $\varepsilon_{0}$ a permissividade do espaço livre, $Z_{0}=\left(\mu_{0} / \varepsilon_{0}\right)^{1 / 2}$ impedância do espaço livre, $\phi$ e $\theta$ coordenadas angulares do sistema de coordenadas esféricas.

A densidade de energia elétrica média total ao redor desta antena é calculada por

$w_{e}^{\text {total }}=\frac{1}{4} \varepsilon_{0}\left(\left|E_{r}\right|^{2}+\left|E_{\theta}\right|^{2}\right)$

Substituindo (3) e (4) em (6) tem-se

$$
\begin{array}{r}
w_{e}^{\text {total }}=\frac{\varepsilon_{0} Z_{0}^{2}|\alpha|^{2}}{4}\left\{\operatorname{sen}^{2} \theta[\right. \\
\left.\quad \frac{1}{(k r)^{2}}-\frac{1}{(k r)^{4}}+\frac{1}{(k r)^{6}}\right]+ \\
\left.+4 \cos ^{2} \theta\left[\frac{1}{(k r)^{4}}+\frac{1}{(k r)^{6}}\right]\right\}
\end{array}
$$

onde $\alpha=k^{2} p_{e} / 4 \pi$. Esta densidade de energia corresponde aos campos de radiação e reativo. Para calcular a densidade de energia somente dos campos reativos $\left(w_{e}\right)$, subtrai-se a densidade de energia correspondente aos campos de radiação $\left(w_{e}^{\text {rad }}\right)$ de (7), ou seja, $w_{e}=w_{e}^{\text {total }}-w_{e}^{\text {rad }}$ [6]. Os campos de radiação, ou seja, aqueles que geram a potência radiada da antena, são [1]

$H_{\phi}^{r a d}=\frac{j \alpha \operatorname{sen} \theta}{k r} e^{-j k r}$ e $E_{\theta}^{r a d}=\frac{j Z_{0} \alpha \operatorname{sen} \theta}{k r} e^{-j k r}$

Desta forma

$$
\begin{aligned}
& w_{e}^{\text {rad }}=\frac{\varepsilon_{0} Z_{0}^{2}|\alpha|^{2}}{4} \frac{\operatorname{sen}^{2} \theta}{(k r)^{2}} \\
& w_{e}=\frac{\varepsilon_{0} Z_{0}^{2}|\alpha|^{2}}{4}\left\{\operatorname{sen}^{2} \theta\left[\frac{1}{(k r)^{6}}-\frac{1}{(k r)^{4}}\right]+\right. \\
& \left.+4 \cos ^{2} \theta\left[\frac{1}{(k r)^{6}}+\frac{1}{(k r)^{4}}\right]\right\}
\end{aligned}
$$

A energia elétrica reativa média $W_{\mathrm{e}}$ ao redor da antena é calculada pela seguinte integral de (10) 


$$
W_{e}=\int_{0}^{2 \pi} \int_{0}^{\pi} \int_{a}^{\infty} w_{e} r^{2} \operatorname{sen} \theta d r d \theta d \phi
$$

Observe que o limite da integração em $r$ é de $a$ ao infinito, visto que foi considerado que não existe energia armazenada dentro da esfera que contém a geometria da antena $(r<a)$. Substituindo (10) em (11) e resolvendo a integral obtém-se

$$
W_{e}=\frac{\mu_{0} k\left|p_{e}\right|^{2}}{24 \pi}\left[\frac{1}{(k a)^{3}}+\frac{1}{k a}\right]
$$

Fazendo o mesmo procedimento para obter (12) mas para agora para o caso da energia magnética reativa média $W_{m}$, tem-se como resultado

$$
W_{m}=\frac{\mu_{0} k\left|p_{e}\right|^{2}}{24 \pi} \frac{1}{k a}
$$

Comparando (12) e (13) observa-se que sempre $W_{e}>W_{m}$ para esta antena, isto quer dizer que esta antena armazena mais energia elétrica do que magnética. Desta observação concluise que a expressão para o cálculo do fator $Q$ é a da primeira linha de (1), a qual é o caso onde $W_{e}>W_{m}$.

A potência radiada desta antena pode ser calculada por

$P^{r a d}=\frac{d W_{e}^{r a d}}{d r} \frac{d r}{d t}=\frac{d W_{e}^{r a d}}{d r} c$

onde $c=1 /\left(\mu_{0} \varepsilon_{0}\right)^{1 / 2}$ é a velocidade da luz no espaço livre. Então a potência radiada é

$P^{r a d}=2 \omega \frac{\mu_{0} k\left|p_{e}\right|^{2}}{24 \pi}$

Substituindo (13) e (15) em (1) obtém-se o fator $Q$ do dipolo elétrico infinitesimal

$Q=\frac{1}{(k a)^{3}}+\frac{1}{k a}$

\section{B. Fator $Q$ da combinação DEI e DMI alinhados}

Neste tipo de combinação os momentos de dipolos elétrico e magnético são respectivamente $\bar{p}_{e}=p_{e} \bar{a}_{z}$ e $\bar{p}_{m}=p_{m} \bar{a}_{z}$. Estes dois dipolos estão localizados na origem do sistema de coordenadas . Considera-se também, como no caso anterior, que esta combinação de dipolos representa uma antena contida em uma circunferência de raio $a$, e que não possua perdas e nem energia reativa armazenada na região $r<a$. Os campos radiados pelo dipolo elétrico são aqueles dados por (2)-(5). Por dualidade, os campos radiados pelo dipolo magnético são

$$
\begin{aligned}
& E_{\phi}=-\frac{j k p_{m} \operatorname{sen} \theta}{4 \pi r}\left(1+\frac{1}{j k r}\right) e^{-j k r} \\
& H_{r}=\frac{p_{m} \cos \theta}{2 Z_{0} \pi r^{2}}\left(1+\frac{1}{j k r}\right) e^{-j k r} \\
& H_{\theta}=\frac{j k p_{m} \operatorname{sen} \theta}{4 Z_{0} \pi r}\left(1+\frac{1}{j k r}+\frac{1}{(j k r)^{2}}\right) e^{-j k r} \\
& E_{r}=E_{\theta}=H_{\phi}=0
\end{aligned}
$$

Utilizando o procedimento da seção anterior, obtém-se a seguinte equação para a energia elétrica reativa

$W_{e}=\frac{k}{24 \pi}\left\{\mu_{0}\left|p_{e}\right|^{2}\left[\frac{1}{(k a)^{3}}+\frac{1}{k a}\right]+\varepsilon_{0}\left|p_{m}\right|^{2}\left[\frac{1}{k a}\right]\right\}$

Definindo a seguinte relação entre os momentos de dipolos $p_{m}=\left(x e^{-j y}\right)^{1 / 2} Z_{0} p_{e}$, onde $x=\left|p_{m}\right|^{2} /\left(Z_{0}^{2}\left|p_{e}\right|^{2}\right)$ é real positivo e $y$ um número real que representa a diferença de fase entre os momentos de dipolo, tem-se a seguinte equação para $W_{e}$

$W_{e}=\frac{\mu_{0} k\left|p_{e}\right|^{2}}{24 \pi}\left[\frac{1}{(k a)^{3}}+(1+x) \frac{1}{k a}\right]$

A potência radiada desta antena e a energia magnética armazenada ao seu redor são respectivamente por

$W_{m}=\frac{\mu_{0} k\left|p_{e}\right|^{2}}{24 \pi}\left[\frac{x}{(k a)^{3}}+(1+x) \frac{1}{k a}\right]$

$P^{r a d}=2 \omega \frac{\mu_{0} k\left|p_{e}\right|^{2}}{24 \pi}(1+x)$

Substituindo (22)-(24) em (1) tem-se o fator de radiação da combinação de um dipolo elétrico e um dipolo magnético alinhados

$Q=\left\{\begin{array}{l}\frac{1}{(1+x)(k a)^{3}}+\frac{1}{k a} \quad \text { se } x<1 \\ \frac{x}{(1+x)(k a)^{3}}+\frac{1}{k a} \text { se } x>1\end{array}\right.$

\section{Fator $Q$ da combinação DEI e DMI ortogonais}

Neste caso os momentos de dipolos são ortogonais e localizados na origem. Os momentos de dipolo são $\bar{p}_{e}=p_{e} \bar{a}_{z}$ e $\bar{p}_{m}=p_{m} \bar{a}_{x}$. A Fig. 1 mostra a localização dos momentos de dipolo para este caso. As considerações feitas para os casos anteriores também são feitas aqui, ou seja, estes dipolos representam uma antena contida dentro de uma esfera fictícia de raio $a$, não existem perdas e não existe energia armazenada dentro da esfera. Os campos radiados pelo dipolo elétrico são aqueles de (2)-(5). Para o dipolo magnético ao longo do eixo 
$x$, os campos radiados são

$$
\begin{aligned}
& E_{\theta}=\frac{j k p_{m} \operatorname{sen} \phi}{4 \pi r}\left(1+\frac{1}{j k r}\right) e^{-j k r} \\
& E_{\phi}=\frac{j k p_{m} \cos \theta \cos \phi}{4 \pi r}\left(1+\frac{1}{j k r}\right) e^{-j k r} \\
& H_{r}=\frac{p_{m} \operatorname{sen} \theta \cos \phi}{2 Z_{0} \pi r^{2}}\left(1+\frac{1}{j k r}\right) e^{-j k r} \\
& H_{\theta}=-\frac{j k p_{m} \cos \theta \cos \phi}{4 Z_{0} \pi r}\left(1+\frac{1}{j k r}+\frac{1}{(j k r)^{2}}\right) e^{-j k r} \\
& H_{\phi}=\frac{j k p_{m} \operatorname{sen} \phi}{4 Z_{0} \pi r}\left(1+\frac{1}{j k r}+\frac{1}{(j k r)^{2}}\right) e^{-j k r} \\
& E_{r}=0
\end{aligned}
$$

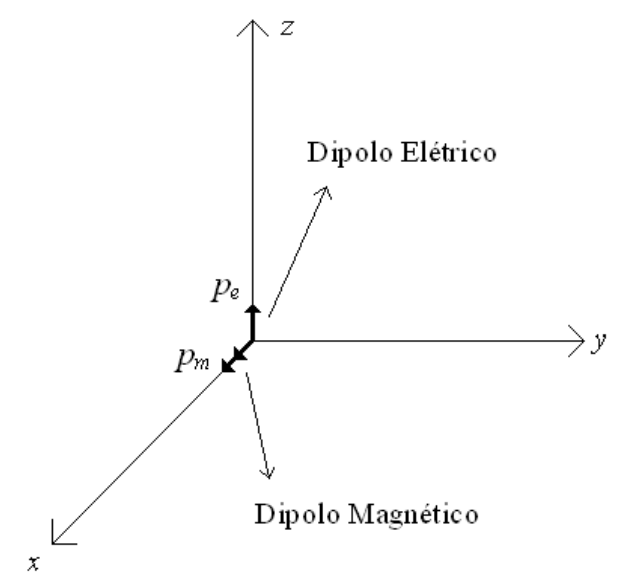

Fig. 1. Combinação de um dipolo elétrico em $z$ e um dipolo magnético em $x$.

Utilizando o mesmo procedimento adotado para os dois casos anteriores, e os campos (2)-(5) e (26)-(31), pode ser mostrado que o fator de qualidade da combinação de dipolos da Fig. 1 é o mesmo do caso do DEI e DMI alinhados da seção anterior, ou seja, o $Q$ da combinação DEI e DMI ortogonais é dado por (25). Uma forma diferente desta equação pode ser dada por

$Q=\left\{\begin{array}{l}\frac{1}{\left[1+\frac{\left|p_{m}\right|^{2}}{Z_{0}^{2}\left|p_{e}\right|^{2}}\right](k a)^{3}}+\frac{1}{k a} \text { se } \frac{\left|p_{m}\right|^{2}}{Z_{0}^{2}\left|p_{e}\right|^{2}}<1 \\ \frac{\left(\frac{\left|p_{m}\right|^{2}}{Z_{0}^{2}\left|p_{e}\right|^{2}}\right)}{\left[1+\frac{\left|p_{m}\right|^{2}}{Z_{0}^{2}\left|p_{e}\right|^{2}}\right](k a)^{3}}+\frac{1}{k a} \text { se } \frac{\left|p_{m}\right|^{2}}{Z_{0}^{2}\left|p_{e}\right|^{2}}>1\end{array}\right.$

\section{RESULTADOS NUMÉRICOS}

Esta seção apresenta alguns cálculos numéricos feitos utilizando (25) e comentários sobre estes resultados.

A Fig. 2 mostra a variação do fator $Q$ dado por (25) em função do parâmetro $x=\left|p_{m}\right|^{2} /\left(Z_{0}^{2}\left|p_{e}\right|^{2}\right)$ para alguns valores de ka. Já a Fig. 3 mostra a variação de Q dado por (25) em função do parâmetro $k a$ para alguns valores de $x=\left|p_{m}\right|^{2} /\left(Z_{0}^{2}\left|p_{e}\right|^{2}\right)$.

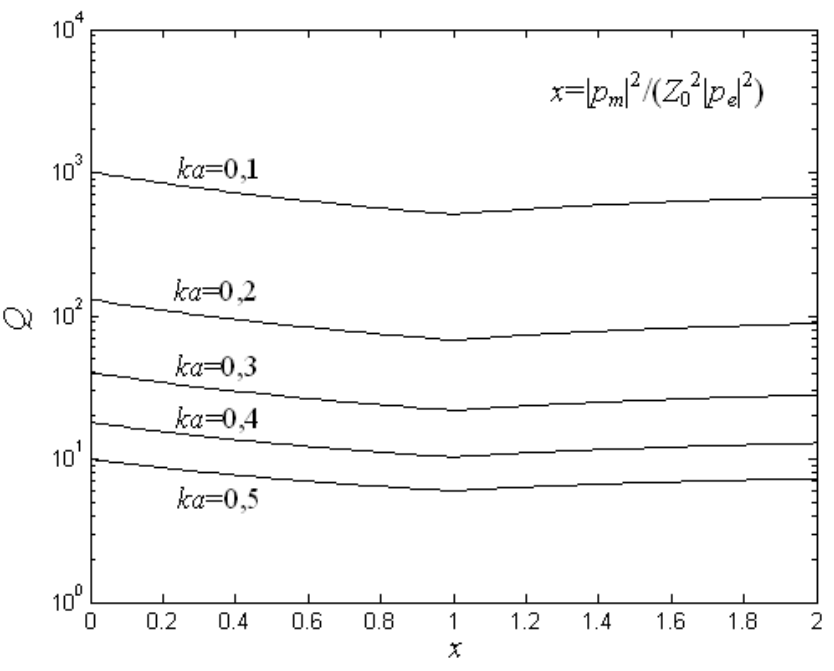

(a)

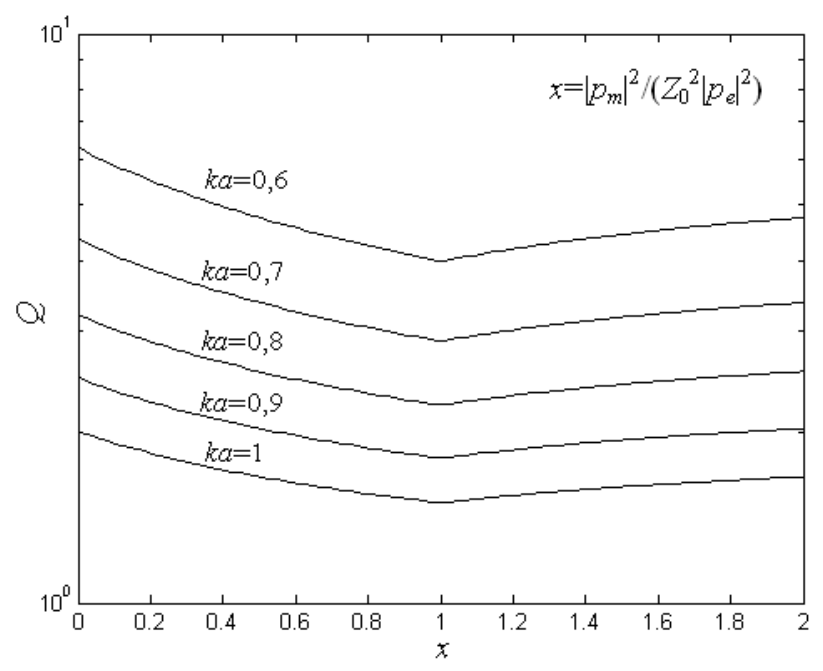

(b)

Fig. 2. Curvas de $Q$ versus $x=\left|p_{m}\right|^{2} /\left(Z_{0}^{2}\left|p_{e}\right|^{2}\right)$ para vários valores de $k a$. (a) $k a=0,1 ; 0,2 ; 0,3 ; 0,4$ e 0,5 . (b) $k a=0,6 ; 0,7 ; 0,8 ; 0,9$ e 1 .

As curvas da Fig. 2 mostram que para cada valor fixo de $k a$, a curva correspondente de $Q$ possui um valor de mínimo em $x=1$, neste ponto tem-se $\left|p_{m}\right|=Z_{0}\left|p_{e}\right|$. Isto quer dizer que os mínimos valores de $Q$ são alcançados quando os momentos de dipolos elétricos e magnéticos possuem amplitudes iguais, independente da diferença de fase e da orientação (dipolos alinhados e ortogonais) entre si. Para $x=1$, a equação (25) torna-se

$Q=\frac{1}{2(k a)^{3}}+\frac{1}{k a}$

Esta equação é o limite fundamental de $Q$ para antenas, ou seja, não existe nenhuma antena que possua uma curva de $Q$ menor ou igual a esta dada por (33). Da Fig. 2 observa-se 
também que a medida que aumenta-se os valores de $k a$ obtém-se valores menores de $Q$.

Das curvas da Fig. 3 observa-se quando se aumenta o valor de $x=0$ até $x=1$, o fator $Q$ diminui até o limite fundamental que é o caso onde $x=1$. A partir de $x=1$, valores maiores de $x$ só aumentarão os valores do fator de radiação $Q$.

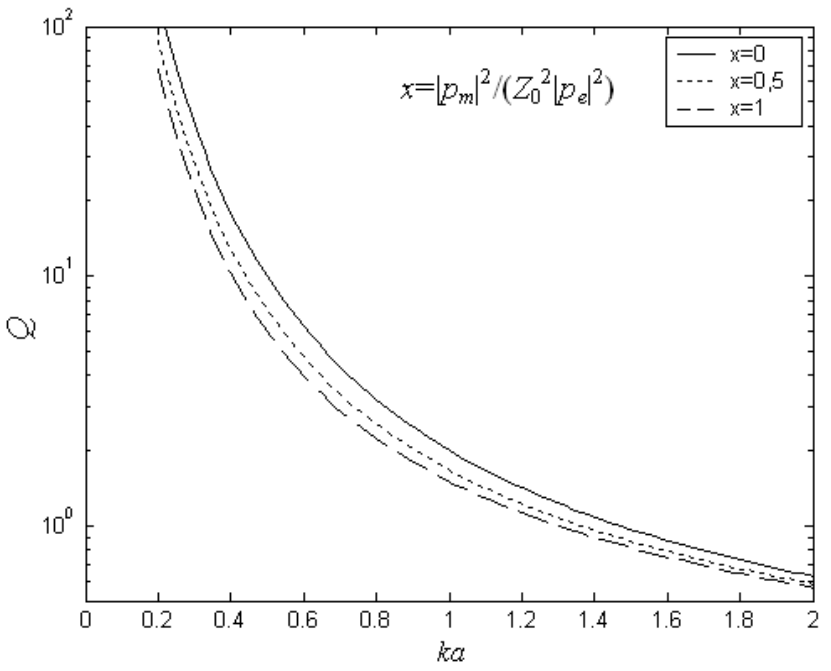

(a)



(b)

Fig. 3. Curvas de $Q$ versus $k a$ para alguns valores de $x=\left|p_{m}\right|^{2} /\left(Z_{0}^{2}\left|p_{e}\right|^{2}\right)$.

(a) $x=0 ; 0,5$ e 1 . (b) $x=1 ; 2$ e 4 .

\section{CONCLUSÕES}

Este trabalho apresentou equações exatas para o cálculo do fator de radiação $Q$ de combinações de dipolos elétricos e magnéticos infinitesimais com diferentes amplitudes, fases e orientações de seus momentos de dipolo. Os dipolos foram colocados no mesmo ponto no espaço livre. As equações do fator $Q$ obtidas para os casos de dipolos alinhados e ortogonais são iguais, isto quer dizer que diferentes orientações de dipolos elétricos e magnéticos podem apresentar os mesmos valores de $Q$. Observou-se também que os fatores de radiação destas combinações não são funções da fase relativa entre os dipolos, isto significa que é possível ter combinações com diferentes configurações de campos radiados, ou seja, diferentes diagramas de radiação para mesmos valores de $Q$.

Da análise apresentada, constatou-se também que o mínimo fundamental de $Q$ é obtido quando os módulos dos momentos de dipolo satisfazem a equação $\left|p_{m}\right|=Z_{0}\left|p_{e}\right|$, onde $Z_{0}$ é a impedância do espaço livre. Este mínimo também é alcançado independente das orientações dos dipolos e da diferença de fase entre os momentos.

Algumas propostas para trabalhos futuros podem ser: análise de diagramas de radiação e ganhos das combinações apresentadas aqui, cálculo do fator $Q$ de combinações de dipolos elétricos e magnéticos infinitesimais localizados em diferentes pontos do espaço, cálculo do fator $Q$ de combinações de dipolos elétricos e magnéticos lineares, e investigar em quais condições estas combinações apresentam mínimos valores de $Q$.

\section{REFERÊNCIAS}

[1] C. A. Balanis, Antenna Theory: Analysis and Design, 3nd ed., Ed. New York: John Wiley, 2005.

[2] H. A. Wheeler, "Fundamental limitations of small antenna", Proc. IRE, vol. 35, pp. 1479-1484, December 1947.

[3] L. J. Chu, "Physical limitations of omni-directional antennas", J. Appl. Phys., vol. 19, pp. 1163-1175, December 1948.

[4] R. E. Collin, S. Rothschild, "Evaluation of antenna Q", IEEE Trans. Ant. Propag., vol. AP-12, pp. 23-27, January 1964.

[5] R. L. Fante, "Quality factor of general ideal antennas", IEEE Trans. Ant. Propag., vol. AP-17, pp. 151-155, March 1969.

[6] J. S. McLean, "A re-examination of the fundamental limits on the radiation Q of electrically small antennas", IEEE Trans. Ant. Propag., vol. 44, N5, pp. 672-675, May 1996.

[7] W. Geyi, P. Jarmuszewski, Y. Qi, "The foster reactance theorem for antennas and radiation Q", IEEE Trans. Ant. Propag., vol. 48, N3, pp. 401-408, March 2000.

[8] W. Geyi, "Physical limitations of antenna", IEEE Trans. Ant. Propag., vol. 51, N8, pp. 2116-2123, August 2003.

[9] W. Geyi, "A method for the evaluation of small antennas Q", IEEE Trans. Ant. Propag., vol. 51, N8, pp. 2124-2129, August 2003.

[10] A. D. Yaghjian, S. R. Best, "Impedance, bandwidth, and $Q$ of antennas", IEEE Trans. Ant. Propag., vol. 53, N4, pp. 1298-1324, April 2005.

[11] K. Q. da Costa, V. Dmitriev, D. C. Nascimento, J. C. da S. Lacava, "Broadband L-probe fed patch antenna combined with passive loop elements", IEEE Ant. Wire. Lett., vol. 6, pp. 100-102, 2007.

[12] K. Q. da Costa, V. Dmitriev, "Combination of electric and magnetic dipoles with single-element feeding for broadband applications", Micro. and Opt. Tech. Let., vol. 48, pp. 8-12, January 2006.

[13] K. Q. da Costa, Victor A. Dmitriev, "A broadband combined (linear and loop) antenna above a ground plane", 2006 IEEE International Workshop on Antenna Technology: Small Antennas and Novel Metamaterials, White Plains, New York, 6 a 8 de Março de 2006.

[14] D.-H. Kwon, "On the radiation $Q$ and the gain of crossed electric and magnetic dipole moments", IEEE Trans. Ant. Propag., vol. 53, N5, pp. 1681-1687, May 2005. 